\title{
Growth Pattern of Silicon Clusters
}

\author{
Atul Bahel, Jun Pan, ${ }^{*}$ and Mushti V. Ramakrishna \\ The Department of Chemistry, New York University, New York, NY 10003-6621. \\ (Mod. Phys. Lett. B 9, XXXX (1995))
}

\begin{abstract}
Tight-binding molecular dynamics simulated annealing technique is employed to search for the ground state geometries of silicon clusters containing 11-17 atoms. These studies revealed that layer formation is the dominant growth pattern in all these clusters. Fullerene-like precursor structures consisting of fused pentagon rings are also observed. The atoms in all these clusters exhibit pronounced preference for residing on the surface.
\end{abstract}

PACS numbers: 36.40.+d, 61.43.Bn, 61.46.+w, 68.35.Bs, 82.65.My

*Also, the Department of Physics, New York University, New York, New York 10003-6621. 
Silicon is a semiconductor with diamond lattice structure. Due to its unique electrical properties it is the most important technological material in electronics industry. As the drive towards miniaturization of electronic devices accelerates, there is a pressing need to investigate the properties of low-dimensional semiconductors $[1-1$. For this reason, the past few years have witnessed enormous scientific activity in the experimental and theoretical investigations of silicon clusters, slabs, and wires of nanometer dimensions «-17.

Since naked silicon clusters are highly reactive, they are mostly synthesized in a molecular beam under high vacuum conditions [5,6]. The number density of available clusters is so low that diffraction based structural investigations are not feasible under these experimental conditions. Consequently, the experimentalists are attempting to infer the structures of these clusters through indirect evidence derived from the reactivities (or sticking coefficients) of these clusters with various reagents. In support of these experimental efforts we have recently started to investigate the structures of silicon clusters using an empirical tightbinding Hamiltonian. The results of these calculations are presented here.

Menon and Subbaswamy have constructed a non-orthogonal tight-binding Hamiltonian for silicon clusters [18, 19]. This method is equivalent in spirit to the well known extended Hückel method [20]. The computational cost of this method is intermediate between the methods based on empirical potential and ab initio molecular orbital techniques. In this method all the valence electrons are explicitly included in the determination of the electronic energy. However, the interaction matrix elements between non-orthogonal atomic orbitals are adjustable parameters. Menon and Subbaswamy have related these matrix elements to Harrison's universal parameters appropriate for the description of bulk Si [21]. In addition, they added four new parameters for the description of the silicon clusters. These additional parameters are derived by fitting to the $\mathrm{Si}_{2}$ bond length and vibrational frequency and to the overall size-dependent cohesive energy curve of clusters [18, 19]. While the parameters are fit to either the experimental or calculated data, none of these parameters are fit to any of the cluster structures. Full details of the Hamiltonian and computational methods are described elsewhere 18,19.

As the size of the cluster grows, the number of structural isomers increases exponentially, with the result that searching the complete configuration space for the global potential energy minimum becomes a formidable task. However, by combining the tight-binding method with the molecular dynamics [22] simulated annealing technique we can efficiently search the cluster configuration space and determine the ground state geometry [23,24]. We used this TB-MD technique in all the calculations reported here.

Figure 1 displays the lowest energy structures obtained using this TB-MD technique for $\mathrm{Si}_{N}$ clusters in the $N=11-17$ atom size range. Figure 2 displays alternate structures obtained for some of these clusters. The $\mathrm{Si}_{11}$ structure consists of a tetragon-pentagon sandwich in the anti-prism geometry with face caps at the bottom and top. This structure may also be viewed as an icosahedron with one missing atom. We also obtained a related structure for $\mathrm{Si}_{11}$ whose cohesive energy is only slightly higher than the global minimum presented in Fig. 1. This local minimum structure, displayed in Fig. 2, consists of a tetragon-tetragon antiprism sandwich with one face cap at the bottom and two face caps at the top. This structure is also one of two possible candidate ground state structures of $\mathrm{Si}_{11}$ found by Rohlfing and Raghavachari using ab initio molecular orbital techniques [25].

The structure of $\mathrm{Si}_{12}$ is similar to $\mathrm{Si}_{11}$, consisting of a pentagon-pentagon anti-prism 
sandwich with face caps at the top and bottom [23,24]. This structure is highly spherical and each atom is five-fold coordinated. Such a spherical cage structure has not been predicted or observed for any other 12-atom elemental cluster. The compound clusters boranes and carboranes are the only other clusters that form icosahedral cage structures.

Adding a face cap to $\mathrm{Si}_{12}$ gives the lowest energy $\mathrm{Si}_{13}$ structure. We also observed an isomer of this cluster, consisting of 4-4-4-1 layers (Fig. 2). The difference in the cohesive energies of these two structures is less than $0.01 \mathrm{eV} /$ atom. We also considered several alternative structures for this cluster, but most of them are either unstable or high energy local minima. For example, placing a $\mathrm{Si}$ atom inside the cage of $\mathrm{Si}_{12}$ yields a high energy local minimum for $\mathrm{Si}_{13}$. Likewise, structures based on hexagon-hexagon sandwiches are found to be unstable.

The $\mathrm{Si}_{14}$ structure is similar to one of the isomers of $\mathrm{Si}_{13}$. It consists of a $4-4-4$ layer structure and two adjacent face caps. By suitably rotating this structure, we may also describe it as a pentagon-pentagon prism sandwich with two caps each at the top and the bottom. The pentagon prism is somewhat distorted and displaced. We also found an isomer of this cluster, consisting of a $\mathrm{Si}_{4}$ subunit riding on top of a $\mathrm{Si}_{10}$ structure (Fig. 2). The cohesive energies of these two structures are within $0.02 \mathrm{eV} /$ atom of each other. This is the first cluster that may be viewed as a super-cluster consisting of stable subunits. We also considered a bi-capped hexagonal anti-prism as a candidate for the ground state of $\mathrm{Si}_{14}$. However, this structure proved to be unstable, indicating that six-atom ring structures are still not favored in these small clusters. Like $\mathrm{Si}_{14}$ cluster, $\mathrm{Si}_{15}$ assumes a layer structure consisting of 1-5-3-5-1 layers. We did not observe any nearly degenerate isomers for $\mathrm{Si}_{15}$.

All the clusters up to $\mathrm{Si}_{15}$ may be described as stacked layers consisting of four- or fivemembered rings. These layers are suitably terminated at the top and bottom. However, $\mathrm{Si}_{16}$ is quite unlike any of these clusters. It is an open cage consisting of fused pentagons, reminescent of the small fullerenes. Since the smallest fullerene must contain at least twenty atoms [26] 28], it appears that $\mathrm{Si}_{16}$ is the precursor to the formation of such fullerene cages [29].

We also found a fullerene-like cage structure for $\mathrm{Si}_{17}$, but it is not the global minimum. Instead, a super-cluster consisting of stable $\mathrm{Si}_{7}$ and $\mathrm{Si}_{10}$ subunits is found to be the global minimum. An alternative super-cluster consisting of $\mathrm{Si}_{6}$ and $\mathrm{Si}_{11}$ subunits is found to be a local minimum (Fig. 2). The structures of these subunits are identical to those of the corresponding isolated clusters [25, 30].

These results indicate that formation of layers is the dominant nucleation and growth pattern in silicon clusters. The exception to this rule is $\mathrm{Si}_{16}$, whose structure is similar to that of small fullerenes consisting of fused pentagon rings. Some clusters seem to prefer to segregate into smaller subunits consisting of 4, 6, 7, or 10 atoms. Each of these subunits is a magic number cluster [30], thus explaining the ability of larger clusters to form superstructures.

In summary, we determined the structures of small silicon clusters through TB-MD simulations. These simulations revealed that $\mathrm{Si}_{11}$ is an incomplete icosahedron, $\mathrm{Si}_{12}$ is a complete icosahedron, $\mathrm{Si}_{13}$ is a surface capped icosahedron, $\mathrm{Si}_{14}$ is a 4-4-4 layer structure with two adjacent face caps, $\mathrm{Si}_{15}$ is a 1-5-3-5-1 layer structure, $\mathrm{Si}_{16}$ is a partially closed cage consisting of fused pentagons reminescent of small fullerenes, and $\mathrm{Si}_{17}$ is a super-structure consisting of $\mathrm{Si}_{7}+\mathrm{Si}_{10}$ subunits. The atoms in all these clusters exhibit a strong preference to lie on 
the surface rather than inside. Formation of stacked layers is the primary mechanism of nucleation and growth in these clusters.

This is the seventh paper in this series on Chemistry of Semiconductor Clusters. This research is supported by the New York University Research Challenge Fund and the Donors of The Petroleum Research Fund (ACS-PRF \# 26488-G), administered by the American Chemical Society. The support of New York University Scientific Visualization Center is gratefully acknowledged. All the computations reported in this paper were carried out on an IBM 580 Workstation. The graphics presented here are generated using the XMol program from the Research Equipment Inc. and the University of Minnesota Supercomputer Center. 


\section{REFERENCES}

$1 \quad$ R. Pool, Clusters: Strange Morsels of Matter, Science 248, 1186 (1990).

2 E. Corcoran, Diminishing Dimensions, Sci. Am. 263, 122 (1990).

$3 \quad$ S. Bjornholm, Contemp. Phys. 31, 309 (1990).

4 R. W. Siegel, Exploring Mesoscopia: The Bold New World of Nanostructures, Physics Today, page 64, October (1993).

5 J. L. Elkind, J. M. Alford, F. D. Weiss, R. T. Laaksonen, and R. E. Smalley, J. Chem. Phys. 87, 2397 (1987); S. Maruyama, L. R. Anderson, and R. E. Smalley, J. Chem. Phys. 93, 5349 (1990); J. M. Alford, R. T. Laaksonen, and R. E. Smalley, J. Chem. Phys. 94, 2618 (1991); L. R. Anderson, S. Maruyama, and R. E. Smalley, Chem. Phys. Lett. 176, 348 (1991).

6 M. F. Jarrold, J. E. Bower, and K. M. Creegan, J. Chem. Phys. 90, 3615 (1989); K. M. Creegan and M. F. Jarrold, J. Am. Chem. Soc. 112, 3768 (1990); M. F. Jarrold, U. Ray, and K. M. Creegan, J. Chem. Phys. 93, 224 (1990); U. Ray and M. F. Jarrold, J. Chem. Phys. 94, 2631 (1991).

7 J. C. Phillips, J. Chem. Phys. 88, 2090 (1988).

8 D. A. Jelski, Z. C. Wu, and T. F. George, Chem. Phys. Lett. 150, 447 (1988).

$9 \quad$ E. Kaxiras, Chem. Phys. Lett. 163, 323 (1989); Phys. Rev. Lett. 64, 551 (1990).

10 B. C. Bolding and H. C. Andersen, Phys. Rev. B 41, 10568 (1990).

11 C. H. Patterson and R. P. Messmer, Phys. Rev. B 42, 7530 (1990).

12 B. L. Swift, D. A. Jelski, D. S. Higgs, T. T. Rantala, and T. F. George, Phys. Rev. Lett. 66, 2686 (1991); D. A. Jelski, B. L. Swift, T. T. Rantala, X. Xia, T. F. George, J. Chem. Phys. 95, 8552 (1991).

13 U. Röthlisberger, W. Andreoni, M. Parrinello, Phys. Rev. Lett. 72, 665 (1994).

14 D. Tomańek and M. Schlüter, Phys. Rev. Lett. 56, 1055 (1986); Phys. Rev. B 36, 1208 (1987); Phys. Rev. Lett. 67, 2331 (1991).

15 M. Menon and K. R. Subbaswamy, Phys. Rev. Lett. 67, 3487 (1991); Int. J. Mod. Phys. B 6, 3839 (1992).

16 M. Menon, K. R. Subbaswamy, and M. Sawtarie, Phys. Rev. B 48, 8398 (1993); Phys. Rev. B 49, 13966 (1994).

17 M. Menon and K. R. Subbaswamy, cpl (xxx: cpl = Chem. Phys. Lett?) 219, 219 (1994).

18 M. Menon and K. R. Subbaswamy, Phys. Rev. B 47, 12754 (1993); Chem. Phys. Lett. 219, 219 (1994).

19 P. Ordejón, D. Lebedenko, and M. Menon, Phys. Rev. B 50, 5645 (1994). We use the first set of parameters with $a=0.08$ and $b=-1.4$ described in this paper.

20 J. P. Lowe, Quantum Chemistry, (Academic Press, New York, 1978).

21 W. A. Harrison, Electronic Structure and the Properties of Solids, (Freeman, San Francisco, 1980).

22 M. P. Allen and D. J. Tildesley, Computer Simulations of Liquids (Oxford University Press, New York, 1987).

23 Atul Bahel and M. V. Ramakrishna, Phys. Rev. B 51, XXXXX (1995).

24 Jun Pan, Atul Bahel, and M. V. Ramakrishna, Surface Review and Letters 2, XXXX (1995).

25 C. M. Rohlfing and K. Raghavachari, Chem. Phys. Lett. 167, 559 (1990). 
26 R. F. Curl and R. E. Smalley, Sci. Am. 10, 54 (1991).

27 W. O. J. Boo, J. Chem. Education 69, 605 (1992).

28 P. W. Fowler, in FULLERENES: Status and Perspectives, Eds. C. Taliani, G. Ruani, and R. Zamboni, (World Scientific, Singapore, 1992).

29 M. V. Ramakrishna and J. Pan, J. Chem. Phys. 101, 8108 (1994); J. Pan and M. V. Ramakrishna, Phys. Rev. B 50, 15431 (1994).

30 K. Raghavachari and V. Logovinsky, Phys. Rev. Lett. 55, 2853 (1985); K. Raghavachari, J. Chem. Phys. 83, 3520 (1985); J. Chem. Phys. 84, 5672 (1986); K. Raghavachari and C. M. Rohlfing, Chem. Phys. Lett. 143, 428 (1988); J. Chem. Phys. 89, 2219 (1988). 


\section{FIGURES}

FIG. 1. The lowest energy structures of $\operatorname{Si}_{N}(N=11-17)$ clusters determined using the tight-binding molecular dynamics simulated annealing technique.

FIG. 2. Alternate structures of $\operatorname{Si}_{N}(N=11,13,14,17)$ found in tight-binding molecular dynamics simulations. The ground state structures of these clusters are displayed in Fig. 1.

FIGURES NOT SUPPLIED 